\title{
Superficie del implante como indicador de riesgo en enfermedades periimplantares
}

\author{
Implant surface as a risk indicator for peri-implant diseases
}

\author{
ARDILA MEDINA CM* \\ GUZMÁN ZULUAGA IC**
}

\begin{abstract}
Ardila Medina CM, Guzmán Zuluaga IC. Superficie del implante como indicador de riesgo en enfermedades periimplantares. Av Periodon Implantol. 2012; 24, 3: 139-144.
\end{abstract}

\section{RESUIMEN}

Las enfermedades periimplantares (EPI) son lesiones inflamatorias que se producen en los tejidos alrededor de los implantes. Diferentes hallazgos clínicos han descrito la importancia de los microorganismos en el desarrollo de estas enfermedades. Los indicadores de riesgo para las EPI incluyen periodontitis, diabetes, factores genéticos, hábito de fumar, consumo de alcohol, higiene oral, ausencia de encía queratinizada y superficie del implante. Se ha demostrado que las características de la superficie del implante pueden influir en la tasa y grado de contacto hueso-implante, especialmente aquellos con superficies rugosas que favorecen una mayor integración ósea cuando se comparan con implantes de superficie lisa. Sin embargo, algunos investigadores han demostrado que pilares de implantes con superficies rugosas tienden a acumular más placa que los de superficie lisa. Bajo este contexto, diferentes superficies de implantes pueden influir sobre la microflora periimplantar y posiblemente sobre el proceso inflamatorio alrededor de los implantes. No obstante, el efecto de la superficie del implante y su diseño, sobre la inflamación de los tejidos alrededor del implante es controvertido.

PALABRAS CLAVE: Inflamación, superficie del implante, periimplantitis, microbiología.

\section{SUMMARY}

The inflammatory lesions that develop in the tissues around implants are collectively recognized as peri-implant diseases. The view that microorganisms play a major role in the development of periimplant diseases is supported by several clinical findings. Indicator factors for peri-implant diseases including periodontitis, diabetes, genetic traits, poor oral hygiene, smoking, alcohol consumption, absence of keratinized mucosa and implant surface. Several studies have demonstrated that implant surface characteristics can influence the rate and degree of bone to implant contact, especially implants with surface roughness that resulted in greater bone integration compared with smooth surface implants. On the other hand, some authors have demonstrated that implant abutments with rough surfaces tended to accumulate more plaque than those with a smooth surface. Under this circumstance, different implant surfaces may influence the peri-implant microbiota and possibly the inflammatory process around implants. However, the effects of various implant designs and surface coatings on peri-implant inflammation have caused controversy.

KEY WORDS: Inflammation, implant surfaces, peri-implantitis, microbiology.

Fecha de recepción: 2 de julio de 2009.

Fecha de aceptación: 10 de julio de 2009.

Profesor Asistente. Facultad de Odontología. Universidad de Antioquía. Miembro de la Junta Directiva de la Asociación Colombiana de Periodoncia y Oseointegración.

** Profesora. Facultad de Odontología. Universidad de Antioquía. Periodoncista. Universidad de Chile. 


\section{INTRODUCCIÓN}

Las lesiones inflamatorias que se desarrollan en los tejidos alrededor de implantes se conocen como enfermedades periimplantares (EPI). Las EPI incluyen dos entidades: mucositis periimplantar (MPI) y periimplantitis (PI). La MPI describe la presencia de inflamación en la mucosa de un implante sin signos de pérdida de soporte óseo (1). Además de la inflamación de la mucosa, la PI se caracteriza por pérdida ósea (1). La MPI ocurre en aproximadamente el $80 \%$ de los sujetos y en el $50 \%$ de los implantes, mientras que la PI sucede entre el $28 \%$ y $56 \%$ de los pacientes y entre el $12 \%$ y $43 \%$ de los implantes (1). Lindhe y cols. (2), demostraron que los tejidos periimplantares y la encía asociada al diente presentan infiltrados inflamatorios de composición similar. La acumulación de bacterias sobre los implantes inician una respuesta inmune en la mucosa periimplantar caracterizada por el establecimiento de una lesión inflamatoria rica en leucocitos y estructuras vasculares (3). Los indicadores de riesgo para EPI incluye periodontitis, diabetes, factores genéticos, hábito de fumar, consumo de alcohol, mala higiene oral, ausencia de encía queratinizada y superficie del implante. Diferentes estudios han propuesto que las características de la superficie del implante pueden influir en la tasa y grado de contacto implante-hueso, especialmente los implantes con superficies rugosas que obtienen mayor integración ósea comparado con los implantes de superficies lisas $(4,5)$. La tabla 1 presenta los diferentes tipos de superficies rugosas en los implantes comercialmente disponibles (6). Abrahamsson y Cardaropoli (7), indicaron que la alteración de la su- perficie del implante amplia su retención al hueso, aumentando la superficie de contacto e incrementando la actividad metabólica de los osteoblastos. Durante el primer año de función del implante, se pierde aproximadamente 1-1,5 mm de hueso marginal alrededor de los implantes, posteriormente, la tasa de pérdida es de $0,1 \mathrm{~mm}$ por año (8). Esto puede ocasionar la exposición de la parte marginal del implante a la microflora bucal, afectando la cantidad y composición de la placa bacteriana en el surco periimplantar (9). Bajo esta contexto, diferentes superficies de implantes pueden influir sobre la microflora periimplantar afectando el proceso inflamatorio alrededor de los tejidos del implante. El objetivo de este artículo es presentar la naturaleza de la reacción inflamatoria en los tejidos periimplantares alrededor de implantes con diferentes clases de superficie. La evidencia científica disponible al respecto se basa principalmente en estudios realizados en animales de experimentación y en muy pocas investigaciones en humanos.

\section{ESTUDIOS EN ANIMALES}

La tabla 2 presenta los estudios en animales que evalúan la asociación entre superficie del implante y enfermedad periimplantar. Berglundh y cols. (10), estudiaron el progreso de periimplantitis (PI) en implantes con diferentes superficies. Se ubicaron tres implantes SLA y tres implantes de superficie pulida en la región de premolares, induciendo PI experimental en cinco perros Beagle. La PI se promovió por medio de ligaduras, permitiendo acumulación de placa bacteriana

\section{TABLA 1.- TIPOS DE SUPERFICIES RUGOSAS EN LOS IMPLANTES COIMERCIALIMENTE DISPONIBLES (6)}

\begin{tabular}{|l|l|c|}
\hline Tipo de rugosidad & Presentación comercial & Grado de rugosidad \\
\hline Leve & $\begin{array}{l}\text { Maquinados } \\
\text { Branemark } 8\end{array}$ & $\mathrm{~S}_{\mathrm{a}} \pm 0,5 \mu \mathrm{m}$ \\
\hline Mediana & $\begin{array}{l}\text { Osseotite } \\
\text { TiUnite } \\
\text { Superficie grabada con chorro de arena (SLA) }\end{array}$ & $\mathrm{S}_{\mathrm{a}}$ entre 1 y $2 \mu \mathrm{m}$ \\
\hline Rugosos & $\begin{array}{l}\text { Plasma-espray (PE) } \\
\text { Superficie con hidroxiapatita (HA) } \\
\text { ITI } \\
\text { Tioblast }\end{array}$ & $\mathrm{S}_{\mathrm{a}}>2 \mu \mathrm{m}$ \\
\hline
\end{tabular}




\section{TABLA 2.- ESTUDIOS EN ANIMALES QUE EVALÚAN LA ASOCIACIÓN ENTRE LA SUPERFICIE DEL IMPLANTE Y ENFERMEDAD PERIIMPLANTAR}

\begin{tabular}{|c|c|c|c|c|}
\hline $\begin{array}{l}\text { Número de } \\
\text { implantes }\end{array}$ & $\begin{array}{l}\text { Implantes evaluados } \\
\text { y pérdida ósea en mm }\end{array}$ & Valor $\mathbf{p}$ & $\begin{array}{l}\text { Tiempo de } \\
\text { evaluación }\end{array}$ & Referencia \\
\hline 30 & SLA $(1,07)$ Pulidos $(0,07)$ & $<0,05$ & 4 meses & 10 \\
\hline 96 & $\begin{array}{l}\text { Maquinados }(1,84) \\
\text { TioBlast }(1,72) \\
\text { SLA }(1,55) \\
\text { TiUnite }(2,78)\end{array}$ & $<0,05$ & 9 meses & 11 \\
\hline 96 & $\begin{array}{l}\text { Maquinados }(3,35) \\
\text { TioBlast }(3,69) \\
\text { SLA }(3,63) \\
\text { TiUnite }(4,44)\end{array}$ & $>0,05$ & 9 meses & 12 \\
\hline 20 & $\begin{array}{l}\text { SLA }(2,5) \\
\text { Superficie lisa }(1,8)\end{array}$ & 0,04 & 7 meses & 13 \\
\hline 36 & $\begin{array}{l}\text { Titanio puro }(2,09) \\
\text { HA }(1,94) \\
\text { PE }(1,79) \\
\text { Grababoacid }(1,62)\end{array}$ & 0,908 & 5 meses & 14 \\
\hline 36 & $\begin{array}{l}\text { Titanio puro }(2,65) \\
\text { HA }(3,26) \\
\text { PE }(2,85) \\
\text { Grababoacid }(3,27)\end{array}$ & $>0,05$ & 5 meses & 15 \\
\hline
\end{tabular}

hasta que se perdiera el $40 \%$ de la altura ósea. Después de cuatro meses se removieron las ligaduras permitiendo acumulación de placa durante cinco meses adicionales. Además de obtener biopsias de los tejidos inflamados, se tomaron radiografías antes y después de presentarse la PI. La mucosa alrededor de todos los implantes se caracterizó por la presencia de un gran infiltrado inflamatorio. La evaluación radiográfica indicó cantidades similares de pérdidas óseas en los dos tipos de superficies, sin embargo, después de la remoción de la ligadura el progreso de la pérdida ósea fue mayor en el implante SLA comparado con el de superficie pulida. Debido a que los implantes usados en la anterior investigación no eran comercialmente disponibles, Albouy y colaboradores (11), analizaron las reacciones tisulares a la formación de placa después de la remoción de las ligaduras en implantes comerciales expuestos a PI experimental. Se realizaron exodoncias de todos los premolares mandibulares de seis perros Labrador. Después de tres meses se instalaron aleatoriamente cuatro implantes, representando cuatro sistemas diferentes de implantes con distintas características de superficie: maquinados, TioBlast, SLA y TiUnite. Después de tres meses se instalaron ligaduras para promover PI experimental. En la semana 12, se removieron las ligaduras, cuando se había perdido cerca del $40-50 \%$ del soporte óseo. La acumulación de placa continuó durante las siguientes 24 semanas. Durante las 36 semanas que duró el experimentó se realizaron exámenes clínicos y radiográficos. En el período activo de la PI se presentó una pérdida ósea de 3,5 y 4,6 mm. La pérdida ósea adicional presentada durante la acumulación de placa después de la remoción de la ligadura $(2,78 \mathrm{~mm})$ fue mayor en los implantes con superficie TiUnite. Las otras marcas presentaron una pérdida ósea de $1,84 \mathrm{~mm}$ (maquinados), 1,72 mm (TioBlast) y 1,55 mm (SLA). El mismo grupo de investigación publicó recientemente los hallazgos histológicos del mismo protocolo de investigación (12), encontrando que todas las muestras ex- 
hibían gran infiltrado celular inflamatorio en los tejidos periimplantares, además de signos de pérdida ósea, corroborando de esta manera los resultados de Berglundh y cols. (10). A pesar de no encontrar diferencias estadísticamente significativas, el estudio histológico mostró mayor gravedad en la PI y en los defectos óseos asociados a los implantes TiUnite, hallazgos ratificados por el estudio clínico y radiográfico realizado por los mismos investigadores, en donde si se encontraron diferencias estadísticamente significativas (11). Otro grupo de investigación brasileño (13), confirmó los resultados obtenidos por Berglundh y cols. (10) al comparar implantes de rugosidad mediana tipo SLA con implantes de superficie lisa. En este estudio, también se extrajeron los premolares derechos e izquierdos de cinco perros Beagle, instalando en un lado implantes SLA y en el otro implantes de superficie lisa. Después de 120 días se conectaron los pilares de cicatrización y se indujo PI experimental mediante ligaduras. Al comparar los dos grupos de implantes se observó una mayor pérdida de inserción clínica en los implantes SLA en el día $90(p=0,04)$.

Contrario a las conclusiones presentadas en las investigaciones descritas anteriormente, existen otros estudios en animales que no han encontrado asociación entre la clase de superficie del implante y el desarrollo de inflamación en enfermedades periimplantares (14-16). Shibli y cols. (14), evaluaron la microflora y la pérdida ósea alrededor de implantes asociada a PI inducida con ligadura. Se colocaron 36 implantes dentales con cuatro superficies distintas ( 9 de titanio puro, 9 HA, 9 PE, 9 grabados con ácido) en mandíbulas edéntulas de seis perros mestizos. Después de la instalación de las ligaduras, se tomaron radiografías y muestras microbiológicas los días $0,20,40$ y 60 . No se encontró diferencia cualitativa en el tipo de bacterias presentes en la PI en ninguna de las superficies evaluadas en los distintos periodos revisados. Igualmente, la pérdida ósea alrededor de los implantes no fue significativamente diferente entre todas las superficies de implantes. Siguiendo el mismo protocolo anterior y utilizando el mismo modelo experimental, Martins y cols. (15), evaluaron la presencia de placa, inflamación de la mucosa alrededor del implante, sangrado al sondaje, profundidad de sondaje, pérdida de inserción clínica, movilidad y pérdida ósea en 36 implantes con cuatro superficies distintas. Los resultados no mostraron diferencias significativas entre las superficies para ninguno de los parámetros estudiados. Finalmente, Pongnarisorn y cols. (16), determinaron la naturaleza del infiltrado inflamatorio asociado con diferentes superficies de implantes en ocho perros Galgos. Se ins- talaron aleatoriamente en cada lado de la mandíbula de los perros, tres implantes experimentales (grabado ácido, maquinado con un surco circunferencial, superficie oxidada) y un implante control (superficie maquinada estándar). A los seis meses se tomaron biopsias gingivales y muestras de placa bacteriana. Los resultados mostraron que el desarrollo de inflamación asociada con implantes es independiente del tipo de superficie, pero relacionado con la presencia de placa bacteriana. Ninguna de las superficies influyó sobre la naturaleza del infiltrado ni sobre la clase de microorganismo encontrado, sin embargo, la presencia de un surco circunferencial alrededor de los implantes maquinados se asoció con grandes infiltrados inflamatorios, debido a la mayor acumulación de placa.

\section{ESTUDIOS EN HUIMANOS}

Muy pocos estudios han examinado la influencia de la superficie del implante como indicador de riesgo para enfermedad periimplantar (17-21). La tabla 3 presenta los estudios en humanos que evalúan la asociación entre la superficie del implante y enfermedad periimplantar.

Ellegaard y cols. (17), evaluaron 31 implantes Astra ${ }^{\circledR}$ y 93 implantes ITI@ en 19 y 56 sujetos, respectivamente. La mayoría de los implantes fueron colocado en la región premolar del maxilar superior y fueron estudiados en un periodo comprendido entre 3 y 84 meses. Durante visitas anuales de mantenimiento, además de tomar radiografías, se revisaron cuatro sitios en los implantes con el fin de evaluar presencia de placa, sangrado y profundidad al sondaje. Fracasaron tres implantes ITI, dos durante el primer año y uno después de 36 meses. Después de cinco años, el 55\% de los implantes ITI permanecieron libres de pérdida ósea. Nuevamente, Ellegaard y colaboradores (18), estudiaron durante cinco años 24 pacientes periodontalmente comprometidos tratados con 80 implantes de diferentes superficies (Astra $®$, Astra $®$ sinus, ITI $®$ y ITI $®$ sinus). Durante el primer año fracasaron un implante Astra $®$ y dos ITI@, y a los 42 meses fracasó un ITI $囚$. A los 36 meses, el $29 \%$ de los implantes ITI $\circledast$ sinus permanecieron libres de una pérdida ósea $\geq 1,5 \mathrm{~mm}$. Baelum y Ellegaard (19), evaluaron dos sistemas de implantes (Astra $\AA$, ITI $®$ ) en pacientes comprometidos periodontalmente, encontrando resultados similares a Ellegaard y colaboradores $(17,18)$. Después de cinco años de observación, el $97 \%$ de los implantes Astra $₫$ y el $94 \%$ de los ITI $®$ estuvieron presentes, sin 


\section{TABLA 3.- ESTUDIOS EN HUMANOS QUE EVALÚAN LA ASOCIACIÓN ENTRE LA SUPERFICIE DEL IMPLANTE Y ENFERMEDAD PERIIMPLANTAR}

\begin{tabular}{|c|c|c|c|c|}
\hline $\begin{array}{l}\text { Número de } \\
\text { implantes }\end{array}$ & $\begin{array}{l}\text { Implantes } \\
\text { evaluados }\end{array}$ & $\begin{array}{l}\text { Implantes perdidos/ } \\
\text { periimplantitis }\end{array}$ & $\begin{array}{l}\text { Tiempo de } \\
\text { evaluación }\end{array}$ & Referencia \\
\hline 124 & $\begin{array}{l}\text { Astra } ® \\
\text { ITI } 尺\end{array}$ & $3 \mathrm{ITI} \circledast$ & 3-84 meses & 17 \\
\hline 80 & $\begin{array}{l}\text { Astra } \circledast \\
\text { Astra } \circledast \text { sinus } \\
\text { ITI } \circledast \\
\text { ITI } \circledast \text { sinus }\end{array}$ & $\begin{array}{l}1 \text { Astra } ® \\
3 \text { ITI } 尺\end{array}$ & 5 años & 18 \\
\hline 459 & $\begin{array}{l}\text { Astra } ® \\
\text { ITI } 尺\end{array}$ & $\begin{array}{l}7 \text { Astra } ® \\
35 \text { ITI } 尺\end{array}$ & 10 años & 19 \\
\hline 73 & $\begin{array}{l}\text { Branemark } ® \\
\text { ITI } 囚\end{array}$ & $3 \mathrm{ITI} \circledast$ & 3 años & 20 \\
\hline 149 & $\begin{array}{l}\text { Maquinados } \\
\text { Tioblast }{ }^{\circledR}\end{array}$ & $\begin{array}{l}1 \text { maquinado } \\
2 \text { Tioblast } \circledR\end{array}$ & 5 años & 21 \\
\hline
\end{tabular}

embargo, solamente el $78 \%$ de los ITI $\circledast$ se mantuvieron después de 10 años. Astrand y cols. (20), realizaron un ensayo clínico con asignación aleatoria para evaluar clínica y radiográficamente, durante tres años, implantes con diferentes superficies (Maquinada: Branemark $®$; y rugosas: ITI®). El estudio incluyó 28 pacientes tratados con 73 implantes maquinados y 77 con superficie rugosa. No se observó periimplantitis en ningún implante maquinado mientras que siete implantes ITI $®$ presentaron este tipo de enfermedad periimplantar.

Contrastando los resultados de los estudios anteriores, Wennstrom y cols. (21), demostraron en un ensayo clínico controlado realizado en 51 pacientes evaluados durante cinco años, que la pérdida ósea no varió entre los implantes maquinados o con superficie rugosa. De hecho, el 78\% de los implantes con superficie maquinada y el $73 \%$ de los implantes con superficie rugosa mostraron menos de $1 \%$ de pérdida ósea durante los cinco años de funcionamiento.

\section{CONCLUSIONES}

La asociación entre la superficie del implante y enfermedades periimplantares está respaldada por evidencia limitada. Los resultados de la mayoría de estudios deben ser evaluados con precaución debido al corto período de evaluación de los estudios, el tamaño de la muestra, el mecanismo de producción de la periimplantitis y el uso de un modelo animal. Se requieren más estudios en humanos, especialmente ensayos clínicos que evalúen a largo plazo el impacto de diferentes tipos de superficies de implantes sobre la enfermedad periimplantar.

\section{BIBLIOGRAFÍA}

1. Zitzmann NU, Berglundh T. Definition and prevalence of peri-implant diseases.J Clin Periodontol 2008;35(Suppl. 8):286-91.

2. Lindhe J, Berglundh T, Ericsson I, Liljenberg B, Marinello C. Experimental breakdown of peri-implant and periodontal tissues. A study in the beagle dog. Clin Oral Implants Res 1992;3:9-16.

3. Zitzmann NU, Abrahamsson I, Berglundh T, Lindhe J. Soft tissue reactions to plaque formation at implant abutments with different surface topography. An experimental study in dogs. J Clin Periodontol 2002;29:456-61.

4. Kim YH, Koak JY, Chang IT, Wennerberg A, Heo SJ. A histomorphometric analysis of the effects of various 
surface treatment methods on osseointegration. Int J Oral Maxillofac Implants 2003;18:349-56.

5. Hallgren C, Sawase T, Ortengren U, Wennerberg A. Histomorphometric and mechanical evaluation of the bone-tissue response to implants prepared with different orientation of surface topography. Clin Implant Dent Relat Res 2001;3:194-203.

6. Albrektsson T, Wennerberg A. Oral implant surfaces: Part l-review focusing on topographic and chemical properties of different surfaces and in vivo responses to them. Int J Prosthodont 2004;17:536-43.

7. Abrahamsson I, Cardaropoli G. Peri-implant hard and soft tissue integration to dental implants made of titanium and gold. Clin Oral Implants Res 2007;18:269-74.

8. Ekelund JA, Lindquist LW, Carlsson GE, Jemt T. Implant treatment in the edentulous mandible: a prospective study on Brånemark system implants over more than 20 years. Int J Prosthodont 2003; 16:602-8.

9. Pongnarisorn NJ, Gemmell E, Tan AES, Henry PJ, Marshall RI, Seymour GJ. Inflammation associated with implants with different surface types. Clin Oral Impl Res 2007; 18:114-25.

10. Berglundh T, Gotfredsen K, Zitzmann NU, Lang NP, Lindhe J. Spontaneous progression of ligature induced periimplantitis at implants with different surface roughness: an experimental study in dogs. Clin Oral Implants Res $2007 ; 18: 655-61$.

11. Albouy JP, Abrahamsson I, Persson LG, Berglundh T. Spontaneous progression of peri-implantitis at different types of implants. An experimental study in dogs I: clinical and radiographic observations. Clin Oral Implants Res 2008;19:997-1002.

12. Albouy JP, Abrahamsson I, Persson LG, Berglundh T. Spontaneous progression of ligatured induced periimplantitis at implants with different surface characteristics. An experimental study in dogs II: histological observations. Clin Oral Implants Res 2009; 20:366-71.

13. Martines RT, Sendyk WR, Gromatzky A, Cury PR. Sandblasted/acid-etched vs smooth-surface implants: implant clinical reaction to experimentally induced periimplantitis in Beagle dogs. J Oral Implantol 2008;34:185-9.
14. Shibli JA, Martins MC, Lotufo RF, Marcantonio E Jr. Microbiologic and radiographic analysis of ligatureinduced peri-implantitis with different dental implant surfaces. Int J Oral Maxillofac Implants 2003;18:383-90.

15. Martins MC, Abi-Rached RS, Shibli JA, Araujo MW, Marcantonio E Jr. Experimental peri-implant tissue breakdown around different dental implant surfaces: clinical and radiographic evaluation in dogs. Int J Oral Maxillofac Implants 2004;19:839-48.

16. Pongnarisorn NJ, Gemmell E, Tan AE, Henry PJ, Marshall RI, Seymour GJ. Inflammation associated with implants with different surface types. Clin Oral Implants Res 2007; 18:114-25.

17. Ellegaard B, Baelum V, Karring T. Implant therapy in periodontally compromised patients. Clin Oral Implants Res 1997;8:180-8.

18. Ellegaard B, Kølsen-Petersen J, Baelum V. Implant therapy involving maxillary sinus lift in periodontally compromised patients. Clin Oral Implants Res 1997;8: 305-15.

19. Baelum V, Ellegaard B. Implant survival in periodontally compromised patients. J Periodontol 2004;75:1404-12.

20. Astrand P, Engquist B, Anzén B, Bergendal T, Hallman M, Karlsson U, et al. A three-year follow-up report of a comparative study of ITI Dental Implants and Brånemark System implants in the treatment of the partially edentulous maxilla. Clin Implant Dent Relat Res 2004; $6: 130-41$

21. Wennstrom JL, Ekestubbe A, Grondahl K, Karlsson S, Lindhe J. Oral rehabilitation with implant-supported fixed partial dentures in periodontitis-susceptible subjects. J Clin Periodontol 2004;31:713-24.

\section{CORRESPONDENCIA}

C. M. Ardila Medina

Carrera 47 No. 20 sur 46

Envigado Antioquía

Colombia

martinardila@gmail.com 